\title{
Normal Pressure Pseudotumor Cerebri: A Series of Six Patients
}

\author{
Mohamed AbdelRahman ABDELFATAH \\ Ain-Shams University, Department of Neurosurgery, Cairo, Egypt
}

\section{ABSTRACT}

AIM: Pseudotumor cerebri (PTC) is a condition characterized by symptoms and signs of increased intracranial pressure (ICP) with no intracranial mass or hydrocephalus, and with normal cerebrospinal fluid (CSF) composition. A variant of PTC known as "Normal Pressure Pseudotumor Cerebri" has the same features of PTC except for normal opening pressure.

MATERIAL and METHODS: This is a series of 6 patients with typical symptoms and signs of increased ICP and normal neuroimaging, but with normal CSF opening pressure. A second lumbar puncture (LP) was done to re-measure the CSF opening pressure.

RESULTS: The mean age of the patients at diagnosis was 25 years (ranging from 19 to 31 years). All patients were female. All patients were obese with a mean body mass index (BMI) of 32.8 (ranging from 30 to 35). The mean duration of symptoms before presentation was 2.8 weeks (ranging from 1 to 8 weeks). The mean CSF opening pressure during the first $\mathrm{LP}$ was $11 \mathrm{~cm} \mathrm{H}_{2} \mathrm{O}$ (ranging from 9 to $15 \mathrm{~cm} \mathrm{H}_{2} \mathrm{O}$ ). The CSF chemistry and culture of all patients were normal. The mean CSF opening pressure in the second LP was $7.3 \mathrm{~cm} \mathrm{H}_{2} \mathrm{O}$ (ranging from 6 to $10 \mathrm{~cm} \mathrm{H}_{2} \mathrm{O}$ ). All patients were symptom free after one week of treatment, but the medications were continued till the complete disappearance of papilledema and the normalization of the visual field. The mean duration of treatment was 8 weeks (ranging from 5 to 12 weeks).

CONCLUSION: Normal pressure PTC should be treated by medical treatment to avoid visual loss.

KEYWORDS: Pseudotumor cerebri, Papilledema, Acetazolamide

\section{INTRODUCTION}

$\mathrm{P}$ seudotumor cerebri (PTC) is a condition characterized by increased intracranial pressure (ICP) with no evidence of intracranial mass or hydrocephalus, and with normal cerebrospinal fluid (CSF) composition (6). PTC patients usually present with typical symptoms and signs of increased ICP, such as headache, vomiting, blurred vision, and papilledema (3).

The neuroimaging abnormalities suggestive of PTC are empty sella, flattening of the posterior aspect of the globe, distension of the perioptic subarachnoid space with or without a tortuous optic nerve, and transverse venous sinus stenosis (3).

ICP is usually measured by lumbar puncture (LP) and a CSF pressure of above $25 \mathrm{~cm} \mathrm{H}_{2} \mathrm{O}$ is one of the diagnostic criteria of PTC (3). Green et al. (4) reported a case of PTC with normal ICP, and introduced the term "Normal Pressure PTC" to describe this variant of PTC. Normal Pressure PTC is rare and written in the literature as case reports $(1,4,8)$.

\section{MATERIAL and METHODS}

This is a series of 6 consecutive patients with normal pressure pseudotumor cerebri whom I experienced from 2007 to 2014 out of 162 patients with PTC. A detailed medical history was taken with general, neurological, and ophthalmological examinations.

Routine labs, brain magnetic resonance imaging (MRI) and magnetic resonance venography (MRV) were done for the six patients. 
Table I: Patients' Demographic Characteristics

\begin{tabular}{ccccccc}
\hline Patients & Age (years) & Sex & Marital state & Pregnant & Body mass index \\
\hline 1 & 24 & Female & Married & In $7^{\text {th }}$ month & Not & 33 \\
\hline 2 & 29 & Female & Divorced & Not & Not & 30 \\
\hline 3 & 19 & Female & Married & Married & In $8^{\text {th }}$ month & 35 \\
\hline 4 & 31 & Female & Married & Not & 33 \\
\hline 6 & 22 & Female & Married & & & \\
\hline
\end{tabular}

Table II: Drug Intake

\begin{tabular}{ccc}
\hline Patients & Excess Vitamin A & Hormonal contraception \\
\hline 1 & No & No \\
\hline 2 & No & No \\
\hline 3 & No & Yes \\
\hline 4 & Yes & Yes \\
\hline 5 & No & No \\
\hline 6 & Yes & No \\
\hline
\end{tabular}

LP was done for all patients in the lateral decubitus position using a yellow $20 \mathrm{G}$ spinal needle under local anaesthesia. The CSF opening pressure (OP) was measured. Only $5 \mathrm{cc}$ CSF were withdrawn. CSF chemistry and culture were done. A second LP was done for all patients few days after the first LP to recheck the CSF opening pressure.

Acetazolamide $500 \mathrm{mg}$ per day was started after the first LP and continued till the complete disappearance of papilledema and the normalization of the visual field.

Patients were followed for at least 12 months after the normalization of the visual field to detect any recurrence of symptoms.

\section{RESULTS}

Six patients are included in this study with a variant of PTC known as "Normal Pressure Pseudotumor Cerebri". This variant comprises $3.7 \%$ from the total number of PTC patients seen in 7 years in Ain-Shams University hospitals. All patients were females in the childbearing years with a mean age at presentation of 25 years (ranging from 19 to 31 years). At the time of presentation, two patients were pregnant in their third trimester, one in the $7^{\text {th }}$ month and the other in the $8^{\text {th }}$ month of gestation.

All patients were obese with a mean body mass index (BMI) of 32.8 (ranging from 30 to 35). Patients' demographic characteristics are shown in Table I. Two patients were taking excess vitamins and especially vitamin A for few months as a hair tonic. Two patients were on hormonal contraception (Table II).
One patient had a history of idiopathic intracranial hypertension 7 years ago and the opening pressure was high $\left(42 \mathrm{~cm} \mathrm{H}_{2} \mathrm{O}\right)$ and resolved on repeated LPs within 6 months.

The six patients presented to the outpatient clinic in Ain-Shams University hospitals with typical symptoms of increased ICP. The mean duration of symptoms before presentation was 2.8 weeks (ranging from 1 to 8 weeks). All patients had headache due to increased ICP, nausea, and blurring of vision.

Three patients were complaining of horizontal diplopia. One patient complained of rapidly progressive diminution of vision within three days (Table III).

On examination, all patients were fully conscious with normal blood pressure. Three patients had bilateral abducens palsy. All patients had papilledema on fundus examination.

Regarding the visual acuity; two patients were 6/6 in both eyes, three were 6/9 in both eyes, and one was hand motion at $30 \mathrm{cms}$ by both eyes.

Visual field examination using Goldmann perimetry revealed that five patients had enlarged blind spot (Table IV).

All patients had iron deficiency anemia with a mean hemoglobin of $9.5 \mathrm{gm} / \mathrm{dl}$ (ranging from 8 to $11 \mathrm{gm} / \mathrm{dl}$ ). All patients had normal glycosylated hemoglobin and normal coagulation profile.

The brain MRI and MRV of all patients were normal except for empty sella that was present in the six patients.

LP was done for all patients by yellow $20 \mathrm{G}$ spinal needle under local anaesthesia. The CSF OP was measured and only 5 cc CSF was withdrawn. The mean CSF OP was $11 \mathrm{cmH}_{2} \mathrm{O}$ (ranging from 9 to $15 \mathrm{cmH}_{2} \mathrm{O}$ ). CSF chemistry and cultures were normal for all patients.

Acetazolamide $500 \mathrm{mg}$ orally daily was started after LP for all patients. All patients complained of low tension positional headache in the first day after LP and improved on bed rest. A second LP was done few days after the first lumbar puncture and the CSF OP was re-checked. The mean CSF OP in the second LP was $7.3 \mathrm{~cm} \mathrm{H}_{2} \mathrm{O}$ (ranging from 6 to $10 \mathrm{~cm} \mathrm{H}_{2} \mathrm{O}$ ) (Table V).

All patients were symptom free after one week of treatment, but acetazolamide was continued till the complete disappearance of papilledema and the normalization of the visual field. 
Table III: Symptoms of the Patients

\begin{tabular}{ccccccc}
\hline Patients & Duration of symptoms & Headache & Nausea & Blurring of vision & Diplopia & Decreased vision \\
\hline 1 & 4 weeks & Present & Present & Present & No & No \\
\hline 2 & 1 week & Present & Present & Present & Present & Yes \\
\hline 3 & 8 weeks & Present & Present & Present & Present & Present \\
\hline 4 & 1 week & Present & Present & Present & Yes & No \\
\hline 5 & 2 weeks & Present & Present & Present & No & Yes \\
\hline 6 & 1 week & Present & Present & Present & & Po
\end{tabular}

Table IV: Signs of the Patients

\begin{tabular}{ccccccc}
\hline Patients & Papilledema & $\begin{array}{c}\text { Visual acuity in } \\
\text { right eye }\end{array}$ & $\begin{array}{c}\text { Visual acuity in } \\
\text { left eye }\end{array}$ & $\begin{array}{c}\text { Visual field in } \\
\text { right eye }\end{array}$ & $\begin{array}{c}\text { Visual field } \\
\text { in left eye }\end{array}$ & $\begin{array}{c}\text { Sixth nerve } \\
\text { palsy }\end{array}$ \\
\hline $\mathbf{1}$ & Present & $6 / 6$ & $6 / 6$ & Enlarged blind spot & Enlarged blind spot & No \\
\hline $\mathbf{2}$ & Present & HM & HM & Not applicable & Not applicable & Bilateral \\
\hline $\mathbf{3}$ & Present & $6 / 9$ & $6 / 9$ & Enlarged blind spot & Enlarged blind spot & Bilateral \\
\hline $\mathbf{4}$ & Present & $6 / 9$ & $6 / 9$ & Enlarged blind spot & Enlarged blind spot & Bilateral \\
\hline $\mathbf{5}$ & Present & $6 / 6$ & $6 / 6$ & Enlarged blind spot & Enlarged blind spot & No \\
\hline $\mathbf{6}$ & Present & $6 / 9$ & $6 / 9$ & Enlarged blind spot & Enlarged blind spot & No \\
\hline
\end{tabular}

HM: Hand motion.

Table V: Opening Pressures (in $\mathrm{cm} \mathrm{H}_{2} \mathrm{O}$ )

\begin{tabular}{|c|c|c|}
\hline Patients & $\begin{array}{c}\text { In First Lumbar } \\
\text { puncture }\left(\mathrm{cm} \mathrm{H}_{2} \mathrm{O}\right)\end{array}$ & $\begin{array}{l}\text { In Second Lumbar } \\
\text { puncture }\left(\mathrm{cm} \mathrm{H}_{2} \mathrm{O}\right)\end{array}$ \\
\hline 1 & 12 & 8 \\
\hline 2 & 10 & 7 \\
\hline 3 & 9 & 7 \\
\hline 4 & 11 & 6 \\
\hline 5 & 15 & 10 \\
\hline 6 & 9 & 6 \\
\hline
\end{tabular}

The mean duration of treatment was 8 weeks (ranging from 5 to 12 weeks).

Weight reduction was strongly recommended for all patients. Excess vitamin A was stopped and anemic patients had iron supplement. Changing the method of contraception from hormonal to another one was advised.

No recurrence of symptoms after 12 months of follow up.

\section{DISCUSSION}

PTC, and its variant "Normal pressure PTC", is an avoidable cause of visual loss. Few diagnostic measures are usually sufficient to determine the correct diagnosis. Most patients will have complete resolution of symptoms without persistent deficits, if they have regular follow-up visits (6).

Repeat lumbar puncture or 24 hours ICP monitoring is suggested in patients who are suspected of having PTC but have a normal ICP by lumbar puncture (2). All patients in this study had repeated LP to re-check the OP. Only 5 cc CSF was withdrawn during the first LP and the second LP was done days later.

If the OP in the second LP was equal or higher than that in the first LP, this means that the OP in the first LP was not the real ICP pressure and this occurred in some cases that were later diagnosed as PTC with high CSF pressure by continuous ICP monitoring. In this study, the OP in the second LP was much lower than that in the first LP in all patients which confirms that the OP in the first LP was correct and so, there was no need for continuous ICP monitoring.

I suggest to reserve continuous ICP monitoring for those cases that had a normal OP in the first LP and a higher OP in the second LP but still lower than $25 \mathrm{~cm} \mathrm{H}_{2} \mathrm{O}$.

The CSF opening pressures in this study does not correlate with the severity of affection of visual acuity or visual field, as the highest CSF OP in the first LP was $15 \mathrm{~cm} \mathrm{H}_{2} \mathrm{O}$ in a patient without affection of visual acuity and with only enlarged blind spots in the visual field, and the patient who presents with severe affection of visual acuity had an OP of $10 \mathrm{~cm} \mathrm{H}_{2} \mathrm{O}$.

Although increased ICP, defined as an opening pressure of over $25 \mathrm{~cm} \mathrm{H}_{2} \mathrm{O}$, is one of the diagnostic criteria of PTC, 
it should be kept in mind that some patients may have optic discs that are more susceptible to lower ICP than others. Even when a patient's ICP is within the normal range, the possibility of PTC should always be considered in a patient with typical clinical features of PTC, such as, papilledema and blind spot enlargement (8).

In this study, two patients were pregnant and two patients were on hormonal contraception, which suggests an underlying hormonal etiology.

The exact cause of the normal CSF pressure at LP in patients with normal pressure PTC despite the symptoms and signs suggestive of increased ICP is not yet known. The presence of papilledema in these patients indicates increased ICP, which due to a certain cause failed to be transmitted to the spinal subarachnoid space.

Obesity has long been associated with the development of PTC. One theory proposes that obesity predisposes patients to having elevated intraabdominal pressure, elevated intrathoracic pressure, and thus elevated central venous pressure as the final common pathway leading to PTC (7). In this study, all patients at presentation were obese with a $\mathrm{BMI}$ ranging from 30 to 35 .

Various systemic diseases and especially anemia have been associated with PTC (2). In this study all patients had iron deficiency anemia.

Johnston et al. reported a series of atypical PTC patients, one of which was a 13-year-old boy whose disc edema rapidly resolved after lumboperitoneal shunt insertion even though his initial CSF pressure was normal (5).

Surgical intervention was not offered to the patients in this study as they rapidly improved with lumbar puncture and acetazolamide, and this was especially significant in the patients with severe visual deficit.
The importance of knowing this variant of PTC is to start the medical treatment soon after lumbar puncture to save vision.

\section{- CONCLUSION}

Normal pressure PTC is a variant of PTC that should be treated by medical treatment to avoid visual loss.

\section{- REFERENCES}

1. Biousse V, Bousser MG, Schaison M: Normal pressure pseudotumor cerebri. J Neuroophthalmol 17(4):279-280, 1997

2. Capriles LF: Intracranial hypertension and iron-deficiency anemia. Arch Neurol 9:147-153, 1963

3. Friedman DI, Liu GT, Digre KB: Revised diagnostic criteria for the pseudotumor cerebri syndrome in adults and children. Neurology 81:1159-1165, 2013

4. Green JP, Newman NJ, Stowe ZN, Nemeroff CB: Normal pressure pseudotumor cerebri. J Neuroophthalmol 16(4): 241-246, 1996

5. Johnston I, Hawke S, Halmagyi M, Teo C: The pseudotumor syndrome. Disorders of cerebrospinal fluid circulation causing intracranial hypertension without ventriculomegaly. Arch Neurol 48: 740-747, 1991

6. Spennato P, Ruggiero C, Parlato RS, Buonocore MC, Varone A, Cianciulli E, Cinalli G: Pseudotumor cerebri. Childs Nerv Syst 27: 215-235, 2011

7. Sugerman HJ, De Maria EJ, Felton WL $3^{\text {rd }}$, Nakatsuka $M$, Sismanis A: Increased intra-abdominal pressure and cardiac filling pressures in obesity-associated pseudotumor cerebri. Neurology 49: 507-511, 1997

8. Suh SY, Kim SJ: IIH with normal CSF pressures? Indian J Ophthalmol 61(11): 681-682, 2013 